\title{
Commitment to glycolysis sustains survival of NO-producing inflammatory dendritic cells
}

\author{
Bart Everts, ${ }^{1,2}$ Eyal Amiel, ${ }^{2}$ Gerritje J. W. van der Windt, ${ }^{1,2}$ Tori C. Freitas, ${ }^{2}$ Robert Chott, ${ }^{1}$ Kevin E. Yarasheski, ${ }^{1}$ \\ Erika L. Pearce, ${ }^{1,2}$ and Edward J. Pearce ${ }^{1,2}$ \\ ${ }^{1}$ Washington University School of Medicine, St Louis, MO; and ${ }^{2}$ Trudeau Institute, Saranac Lake, NY
}

\begin{abstract}
TLR agonists initiate a rapid activation program in dendritic cells (DCs) that requires support from metabolic and bioenergetic resources. We found previously that TLR signaling promotes aerobic glycolysis and a decline in oxidative phosphorylation (OXHPOS) and that glucose restriction prevents activation and leads to premature cell death. However, it re-
\end{abstract}

mained unclear why the decrease in OXPHOS occurs under these circumstances. Using real-time metabolic flux analysis, in the present study, we show that mitochondrial activity is lost progressively after activation by TLR agonists in inflammatory blood monocyte-derived DCs that express inducible NO synthase. We found that this is because of inhibi- tion of OXPHOS by NO and that the switch to glycolysis is a survival response that serves to maintain ATP levels when OXPHOS is inhibited. Our data identify $\mathrm{NO}$ as a profound metabolic regulator in inflammatory monocyte-derived DCs. (Blood. 2012;120(7):1422-1431)

\section{Introduction}

Dendritic cells (DCs) express TLRs that allow them to detect and respond to pathogen-derived molecules. ${ }^{1,2}$ In response to TLR agonists, DCs transition from a resting state to an activated state through a process that that involves the induction of expression of genes encoding a broad array of proteins such as cytokines, chemokines, and costimulatory molecules. ${ }^{3}$ Activated DCs play a central role in orchestrating the development of immune responses.

Recently, we showed that after exposure to TLR agonists, DCs undergo a striking metabolic transition evident as a pronounced increase in the glycolytic rate. ${ }^{4}$ This is highly reminiscent of Warburg metabolism, ${ }^{5}$ in which tumor cells preferentially use glycolysis rather than catabolic mitochondrial pathways to conserve and generate metabolic resources to meet the demands of cellular proliferation while still producing sufficient ATP to permit these processes to occur. ${ }^{6,7}$ Moreover, the increase in glycolytic rate in DCs was found to be dependent on the PI3K/Akt pathway, ${ }^{4}$ which is one of the most commonly mutated signaling pathways in tumors. ${ }^{8}$ We reasoned that glycolysis could serve essentially the same purpose in active DCs as it is thought to do in tumors. ${ }^{4}$ This view was supported by the fact that glucose restriction inhibits severely the activation and life span of DCs exposed to TLR agonists. ${ }^{4}$ However, unlike in most cancers, which continue to consume oxygen at rates comparable to normal tissues despite increased glycolytic rates, ${ }^{9}$ activated DCs use significantly less oxygen than do resting DCs. ${ }^{4}$ Thus far, the molecular mechanisms underlying mitochondrial impairment in activated DCs, and the metabolic consequences of the loss of mitochondrial function, remain unclear.

To address these issues, we have in the present study, undertaken a detailed analysis of mitochondrial function in DCs after TLR stimulation. Using extracellular flux analysis to measure changes in oxygen consumption in real time, we found that 6 hours after stimulation, mitochondrial oxygen consumption was progressively lost due to the initiation of NO production. By 24 hours after exposure to TLR agonists, mitochondria in NO-producing DCs exhibit no evidence of oxidative phosphorylation (OXPHOS) or of an active tricarboxylic acid (TCA) cycle. We show that the switch to sustained glycolytic metabolism by activated DCs in vitro and monocyte-derived inflammatory DCs (moDCs) in vivo is a response to the inhibition of respiration by NO and is essential for DC function and survival because it provides essential ATP in the absence of a functioning respiratory chain.

\section{Methods}

Mice

Wild-type, inducible NO synthase-knockout $\left(i N O S^{-1-}\right)$, ovalbuminspecific CD4 (OT-II)-, and or CD8 (OT-I)-transgenic C57B1/6 mice were purchased from The Jackson Laboratory, and re-derived stocks were maintained at the Trudeau Institute and Washington University (St Louis, MO) under specific pathogen-free conditions under protocols approved by the institutional animal care and use committee.

\section{In vitro DC differentiation and isolation of splenic DCs}

BM-derived DCs were generated as described previously..$^{10}$ Briefly, BM cells were differentiated in the presence of GM-CSF $(20 \mathrm{ng} / \mathrm{mL})$ in complete DC medium (RPMI containing 10\% FCS, $100 \mathrm{U} / \mathrm{mL}$ of penicillin/ streptomycin, and $2 \mathrm{mM}$ L-glutamine) for 6 days. Inflammatory moDCs were elicited in vivo by infecting mice intravenously with a sublethal dose of $2 \times 10^{5}$ CFUs of the attenuated $\triangle$ ActA strain of Listeria monocytogenes. ${ }^{11}$ One or 2 days after infection, spleens were harvested and digested at $37^{\circ} \mathrm{C}$ for 20 minutes in RPMI with collagenase $\mathrm{D}(1 \mathrm{mg} / \mathrm{mL}$; Roche $)$ in the presence of DNAse I ( $20 \mu \mathrm{g} / \mathrm{mL}$; Sigma-Aldrich). Spleens were put through $70-\mu \mathrm{m}$ strainers to generate single-cell suspensions and RBCs were
Submitted March 23, 2012; accepted June 27, 2012. Prepublished online as Blood First Edition paper, July 11, 2012; DOI 10.1182/blood-2012-03-419747.

The online version of this article contains a data supplement.
The publication costs of this article were defrayed in part by page charge payment. Therefore, and solely to indicate this fact, this article is hereby marked "advertisement" in accordance with 18 USC section 1734.

(C) 2012 by The American Society of Hematology 
lysed with ACK lysis buffer $\left(0.15 \mathrm{M} \mathrm{NH} \mathrm{N}_{4} \mathrm{Cl}, 1 \mathrm{mM} \mathrm{KHCO}{ }_{3}\right.$, and $0.1 \mathrm{mM}$ EDTA). Where indicated, 1-day L monocytogenes-infected mice were injected intraperitoneally with PBS or 2-deoxyglucose (2-DG; $4 \mathrm{~g} / \mathrm{kg}$ ) and 6 hours later spleens were harvested for analysis of DC subsets. Inflammatory moDCs were tracked and isolated from splenocytes based on CD11c, MHC-II (H2-IA/E $\left.{ }^{b}\right)$, Ly6C $^{\text {hi }}$, and CD11 bint expression. Two resident DC populations were isolated from single-cell suspensions prepared from spleens of naive mice based on CD11c, MHC-II (H2-IA/E $)$, and either CD11b and CD4 or DEC205 and CD8 $\alpha$ expression. All in vivo DC populations were isolated using an influx sorter (BD Biosciences).

\section{DC culture}

Day 6 BM-derived DCs or isolated splenic DCs were washed in complete DC medium and, where indicated, pulsed with the TLR4 agonist lipopolysaccharide (LPS; Escherichia coli serotype 0111:B4,;100 ng/mL; SigmaAldrich); TLR1/2 agonist Pam3CSK4 (1 $\mu \mathrm{g} / \mathrm{mL})$, TLR3 agonist poly(I:C) $(10 \mu \mathrm{g} / \mathrm{mL})$, TLR7/8 agonist R848 $(1 \mu \mathrm{g} / \mathrm{mL})$, and TLR9 agonist CpG $(5 \mu \mathrm{g} / \mathrm{mL}$; all InvivoGen); rTNF- $\alpha(10 \mathrm{ng} / \mathrm{mL}), \mathrm{rIFN}-\gamma(10 \mathrm{ng} / \mathrm{mL}$; both PeproTech) in the presence or absence of NOS inhibitor S-ethyl-isothiourea (SEITU, $500 \mu \mathrm{M}$; Cayman Chemical). In experiments in which glucose in the medium was replaced by galactose, glucose-free RPMI and dialyzed FCS were used to ensure the absence of glucose in the culture media.

\section{T-cell proliferation assay}

DCs were treated for 6 hours with LPS in the presence or absence of ovalbumin $(1 \mathrm{mg} / \mathrm{mL}$ of endotoxin-free egg white prepared in the laboratory) and washed. Then, $2 \times 10^{4}$ pulsed DCs were cultured with $2 \times 10^{5}$ CFSE-labeled CD62L $\mathrm{L}^{\text {hi }} \mathrm{CD} 44^{\text {lo }}$ naive OT-II or OT-I T cells. T-cell proliferation was assessed by CFSE dilution by FACS.

\section{Metabolism assays}

Glucose and lactate levels in the medium were measured using a glucose assay kit and a lactate assay kit (Eton Bioscience) per the manufacturer's instructions. For real-time analysis of the extracellular acidification rate (ECAR) and the oxygen consumption rate (OCR), DCs were analyzed using an XF-24 and XF-96 Extracellular Flux Analyzer (Seahorse Bioscience). In brief, DCs were plated in XF-24 or XF-96 cell culture plates $\left(2 \times 10^{5}\right.$ cells/well in $200 \mu \mathrm{L}$ or $7 \times 10^{4}$ cells/well in $\left.70 \mu \mathrm{L}\right)$ and either left unstimulated or stimulated with indicated TLR ligands. At the indicated time points, DCs were washed and analyzed in XF Running Buffer (unbuffered RPMI, 10mM glucose, 10\% FCS, $100 \mathrm{U} / \mathrm{mL}$ penicillin/ streptomycin, $2 \mathrm{mM} \mathrm{L-glutamine,} \mathrm{and} 20 \mathrm{ng} / \mathrm{mL}$ of GM-CSF) per the manufacturer's instructions to obtain real-time measurements of OCR and ECAR. Where indicated, ECAR and/or OCR were analyzed in response to $1 \mu \mathrm{M}$ oligomycin, $1.5 \mu \mathrm{M}$ fluoro-carbonyl cyanide phenylhydrazone (FCCP) and $100 \mathrm{nM}$ rotenone plus $1 \mu \mathrm{M}$ antimycin A (all Sigma-Aldrich); $500 \mu \mathrm{M}$ SEITU, $250 \mu \mathrm{M}$ NO donor S-nitroso-N-acetylpenicillamine (SNAP; Invitrogen), $50 \mathrm{ng} / \mathrm{mL}$ of phorbol 12-myristate 13-acetate (Sigma-Aldrich) or $10 \mu \mathrm{M}$ NADPH oxidase inhibitor apocynin (Sigma-Aldrich). For ATP analysis, DCs were treated as indicated for 24 hours, after which time cells were washed and resuspended in $100 \mu \mathrm{L}$ of PBS. Samples were boiled for 5 minutes and relative ATP levels were determined using an ATP determination kit (Invitrogen) per the manufacturer's instructions. For glucose-tracing experiments, DCs were stimulated for 21 hours with LPS or left unstimulated, followed by 3 hours of pulse labeling with ${ }^{13} \mathrm{C}_{6}$-glucose (Cambridge Isotope Laboratories) or natural ${ }^{12} \mathrm{C}$-glucose, and intracellular metabolites were extracted by washing in ice-cold methanol as described previously. ${ }^{12} \mathrm{Next}, 200 \mu \mathrm{L}$ of methanol extract was evaporated to dryness and treated with $80 \mu \mathrm{L}$ of N,O-bis(trimethylsilyl) trifluoroacetamide (10\% trimethylchlorosilane; Regis) and heated at $70^{\circ} \mathrm{C}$ for 1 hour to convert the metabolites to their trimethylsilyl derivatives. Authentic citrate was purchased (Sigma-Aldrich), converted to the trimethylsilyl form, and used to determine gas chromatography retention times and its positive chemical ionization fragmentation pattern. Derivatized standards and samples were injected $(2 \mu \mathrm{L})$ onto a DB-XLB $(30 \mathrm{~m}, 0.25 \mathrm{~mm}$ internal diameter, $0.25-\mu \mathrm{m}$ film) column (Agilent Technologies) and nominal mass/charge $(\mathrm{m} / \mathrm{z})$ for molecular and fragment ions were monitored using a mass selective detector (6890 gas chromatograph and 5973 quadrupole mass spectrometer; Agilent Technologies). Selected ion monitoring was used to quantify $\mathrm{m} / \mathrm{z}$ signal intensities for citrate: $465-471$. Signal intensities $\left({ }^{13} \mathrm{C}\right.$ species/natural ${ }^{12} \mathrm{C}$ species) for citrate were calculated and compared among experimental conditions.

\section{Flow cytometry}

Fluorochrome-conjugated Abs for surface staining for FACS analysis and 7-amino-actinomycin D were purchased from BD Biosciences except for anti-CD11c and anti-CD40, which were obtained from eBiosciences. For intracellular staining of iNOS, DCs were fixed in $4 \%$ ultrapure paraformaldehyde, stained for 1 hour at room temperature with mouse anti-NOS2 (clone C-11; Santa Cruz Biotechnology) and rat anti-mouse IgG1-APC (BD Biosciences) in $0.2 \%$ saponin buffer. Mitochondrial membrane potential was determined using $20 \mu \mathrm{M} 3,3^{\prime}$-dihexyloxacarbocyanine iodide (DiOC 6 ; Invitrogen) and superoxide $\left(\mathrm{O}_{2}{ }^{-}\right)$production using $1 \mu \mathrm{M}$ dihydroethidium (Sigma-Aldrich) in the FITC and PE channels, respectively, after 20 minutes of incubation at $37^{\circ} \mathrm{C}$. Cytokine concentrations in supernatants of 24-hour TLR-stimulated DCs were determined using cytometric bead array (BD Biosciences) according to the manufacturer's instructions. Samples were analyzed on a FACSCanto II flow cytometer (BD Biosciences).

\section{RT-PCR and mtDNA/nDNA quantification}

RNA isolations were done using the RNeasy kit (QIAGEN) and singlestrand cDNA was synthesized using a high-capacity cDNA Reverse Transcription Kit (Applied Biosystems). Primers were purchased from Applied Biosystems and real-time PCR was performed by the TaqMan method using an Applied Biosystems 7500 sequence detection system. The expression levels of mRNA were normalized to the expression of a housekeeping gene ( $\beta$-actin). To determine mitochondrial DNA (mtDNA)/nuclear DNA (nDNA) ratios, genomic DNA was extracted using the phenol-chloroform method. Primers for CO1 (forward: 5'-TGCTAGCCGCAGGCATTAC-3' and reverse: $5^{\prime}$-GGGTGCCCAAAGAATCAGAAC-3') and NDUFV1 (forward: 5'-CTTCCCCACTGGCCTCAAG-3' and reverse: 5'-CCAAAACCCAGTGATCCAGC- $3^{\prime}$ ) were used to quantify mtDNA and nDNA, respectively. ${ }^{13}$ Real-time PCR was performed based on SYBR Green using an Applied Biosystems 7500 sequence detection system.

\section{Statistical analysis}

Data were analyzed using Prism Version 5 software (GraphPad). Comparisons for 2 groups were calculated using unpaired 2-tailed Student $t$ tests. A 2-way ANOVA with a Bonferroni posttest was used for the glucosetracing experiment. Differences were considered significant at $P<.05$.

\section{Results}

\section{Changes in mitochondrial function in DCs after TLR activation}

Our previous work indicated that OXPHOS was significantly decreased in BM-derived DCs at 24 hours after exposure to TLR agonists. ${ }^{4}$ To determine when after activation this decline in mitochondrial activity occurs, we used extracellular flux analysis to perform detailed measurements of mitochondrial OCR over time after exposure to the TLR4 ligand LPS. These analyses revealed that OCR did not decrease immediately after TLR stimulation, but instead was slightly increased during the first 6 hours after TLR ligation, after which a pronounced decline in OCR was observed that was sustained until at least 24 hours (Figure 1A). A detailed analysis of OCR in DCs at 24 hours after activation revealed the extent of mitochondrial loss of function. Whereas resting DCs showed characteristic changes in OCR in response to inhibition of the mitochondrial ATP-synthase by oligomycin, uncoupling of OXPHOS from ATP synthesis by FCCP and inhibition of the 
A

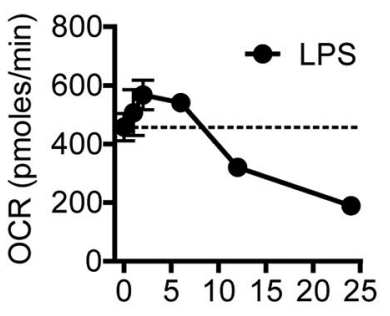

time after stimulation (h)

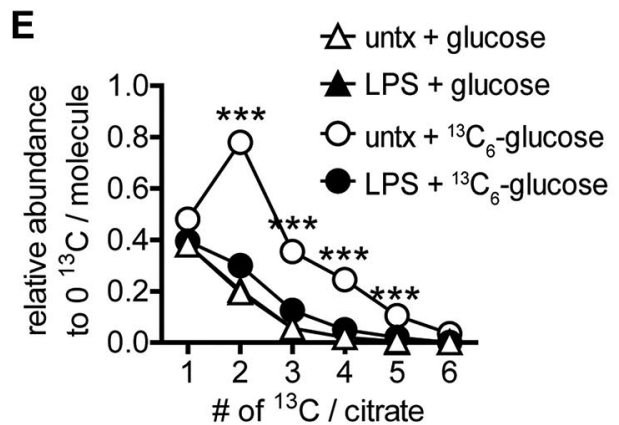

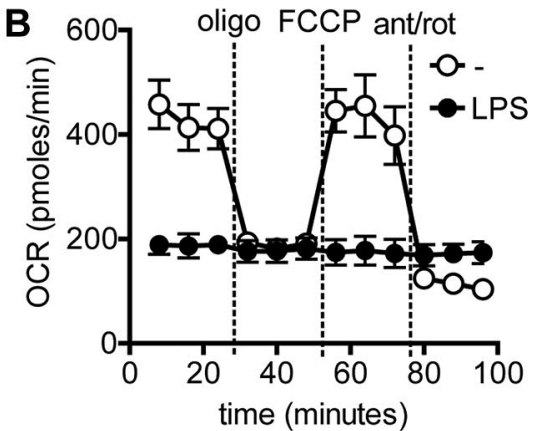

$\mathbf{F}$

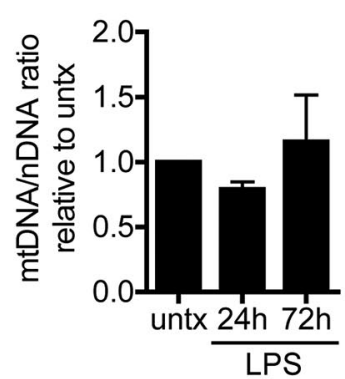

C

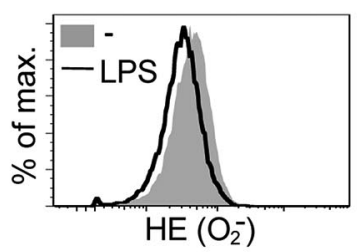

D

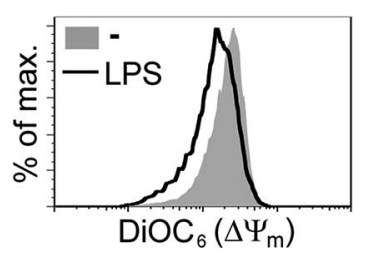

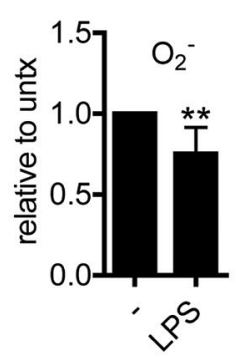

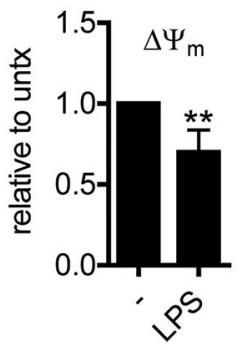

Figure 1. Changes in mitochondrial function in DCs after TLR activation. (A) BM-derived DCs were seeded in a Seahorse XF-24 analyzer, stimulated with LPS, and at the indicated time points OCR was determined. Dashed line represents baseline OCR. Data represent means \pm SD of triplicates. One representative experiment of 3 is shown. (B) DCs were seeded in a Seahorse XF-24 analyzer, stimulated with medium as a control or LPS for 24 hours, and real-time OCR was determined during sequential treatments with oligomycin (ATP-synthase inhibitor), FCCP, and antimycin-A/rotenone (ETC inhibitors). Data represent means \pm SD of triplicates. One of 6 experiments is shown. $(C-D)$ Twenty-four-hour LPS-stimulated DCs were stained with hydroethidin $(\mathrm{HE})$ to detect $\mathrm{O}_{2}{ }^{-}$production or $\mathrm{DiOC}_{6}$ to determine mitochondrial membrane potential $\left(\Delta \Psi_{\mathrm{m}}\right)$. A representative histogram is shown and the bar graph represents means \pm SD of 3 independent experiments. ${ }^{*} P<.01$. (E) Twenty-one hours after stimulation with LPS or medium, DCs were fed ${ }^{13} \mathrm{C}_{6}$-glucose or normal ${ }^{12} \mathrm{C}$-glucose (both $10 \mathrm{mM}$ ) for 3 hours. Next, metabolites were isolated from cells by methanol extraction and the ${ }^{13} \mathrm{C}$ content in citrate was quantified by mass spectrometry. Amounts of citrate carrying $1-6{ }^{13} \mathrm{C}$ are shown relative to levels of citrate harboring no ${ }^{13} \mathrm{C}$ (only ${ }^{12} \mathrm{C}$ ). Relative amounts of naturally occurring ${ }^{13} \mathrm{C}$ in citrate when DCs are pulsed with unlabeled glucose are shown in triangles. Data represent means \pm SD of triplicates. ${ }^{* \star \star} P<.001 \mathrm{compared}$ with naturally occurring ${ }^{13} \mathrm{C}$ in citrate. Data represent means $\pm \mathrm{SD}$ of triplicates of 1 experiment. $(\mathrm{F}) \mathrm{mtDNA} / \mathrm{nDNA}$ ratio at different time points after LPS stimulation. Data represent means \pm SD of 2 independent experiments. ant/rot indicates antimycin-A/rotenone; and oligo, oligomycin.

electron transport chain (ETC) by antimycin-A/rotenone (explained in supplemental Figure 1, available on the Blood Web site; see the Supplemental Materials link at the top of the online article), DCs that had been activated for 24 hours by LPS were entirely nonresponsive to these drugs (Figure 1B). Consistent with these observations, $\mathrm{O}_{2}{ }^{-}$production (Figure $1 \mathrm{C}$ ) and mitochondrial membrane potential ( $\Delta \Psi_{\mathrm{m}}$; Figure 1D), indicators of ETC use, ${ }^{14}$ were significantly reduced in these cells. This phenomenon was not specific to TLR4-activated DCs, because other TLR agonists (with the exception of the TLR1/2 agonist Pam3cys) induced similar declines in mitochondrial respiration in these DCs (supplemental Figure 2A).

OXPHOS is fueled by the TCA cycle. To determine whether a loss in mitochondrial respiration was paralleled by an impairment in function of the TCA cycle, activated DCs were cultured in the presence of ${ }^{13} \mathrm{C}_{6}$-glucose as their only glucose source, and the accumulation of ${ }^{13} \mathrm{C}$ in citrate, the first TCA-cycle intermediate to receive glycolysis-derived carbon through acetyl-CoA, ${ }^{15}$ was determined using gas chromatography-mass spectrometry. In resting DCs, citrate was highly enriched for ${ }^{13} \mathrm{C}$ compared with control DCs cultured with natural ${ }^{12} \mathrm{C}$-glucose (Figure $1 \mathrm{E}$ ). However, ${ }^{13} \mathrm{C}$ incorporation into citrate in 24-hour LPS-activated cells was indistinguishable in cells cultured with ${ }^{13} \mathrm{C}_{6}$-glucose versus natural ${ }^{12} \mathrm{C}$-glucose, indicating that the flow of glucose carbon through the TCA cycle was halted in LPS-activated DCs. These data are consistent with the decrease in OCR and mitochondrial membrane potential in activated DCs, and support the view that TLRsignaling results in a profound decline in mitochondrial activity.
The striking decline in mitochondrial processes in DCs that had been activated with LPS for 24 hours raised the possibility that these cells had cleared their mitochondria by mitophagy. ${ }^{16}$ However, the ratio of mtDNA to nDNA was equivalent in resting and activated DCs (Figure 1F), indicating that LPS activation did not reduce the number of mitochondria per cell. These data indicate that after an early increase in mitochondrial respiration during the first 6 hours, TLR-mediated activation of DCs leads to a persistent and profound collapse in mitochondrial function that is characterized by the absence of a functional ETC and glucose-carbon flux through the mitochondrial TCA cycle.

\section{TLR-driven impairment of mitochondrial respiration in DCs is due to functional inhibition by iNOS-derived NO}

Nonmitochondrial OCR, revealed by treatment with antimycinA/rotenone, was higher in LPS-activated cells than in resting DCs (Figure 1B). Outside of the mitochondrion, oxygen can be consumed in the generation of reactive oxygen species and NO by NADPH oxidase and NOS, respectively. ${ }^{17,18}$ Because reactive oxygen species and $\mathrm{NO}$ have been reported to inhibit mitochondrial function in other systems, ${ }^{19,20}$ we investigated whether these processes could also play a role in this situation. Although NAPDH oxidase was functionally expressed in BM-derived DCs ${ }^{21}$ (Figure 2A), specific inhibition of this enzyme complex had no effect on nonmitochondrial oxygen consumption (Figure 2B), suggesting that this enzyme is not active and does not play a role in impairment of mitochondrial function in these cells. With regard to NOS, consistent with previous reports, ${ }^{22}$ NOS 2 mRNA (Figure 2C) and 
A

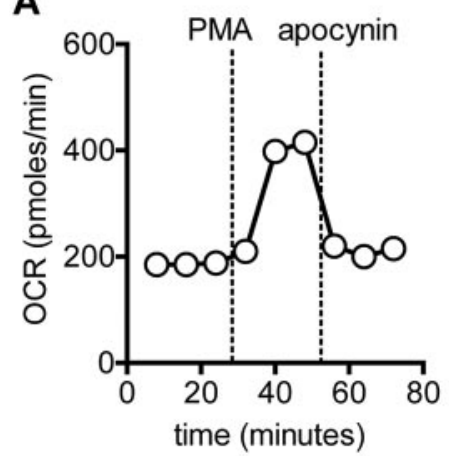

E

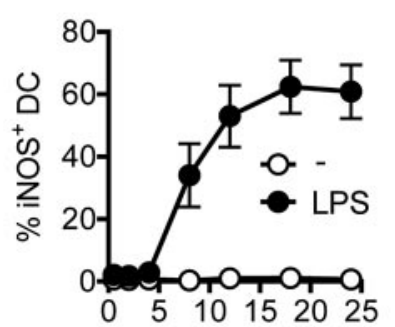

time after stimulation (h)
B

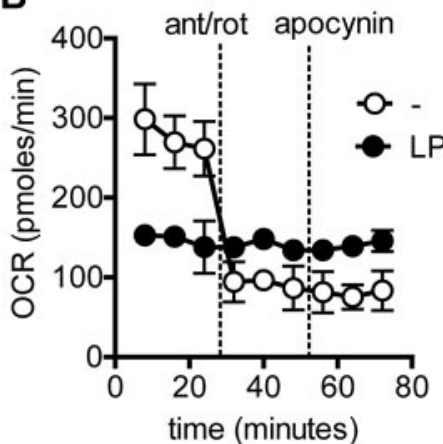

F

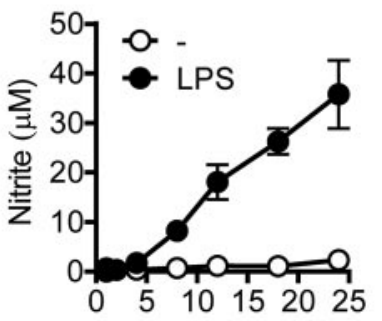

time after stimulation (h)
C

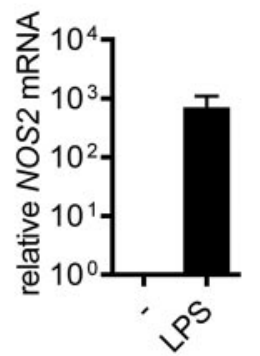

D

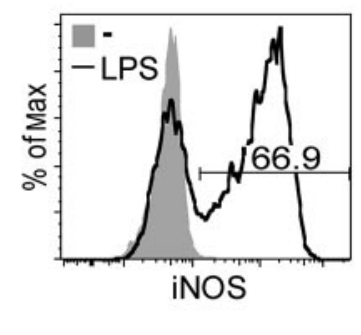

G

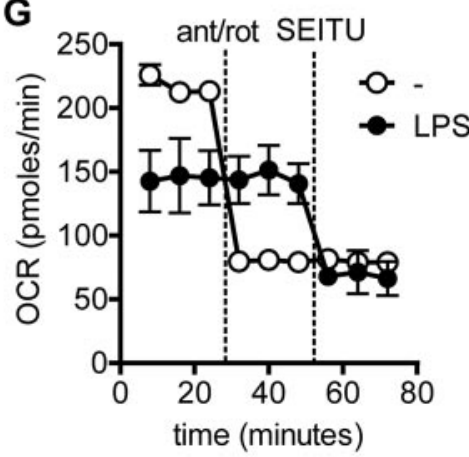

Figure 2. Activation of DCs by LPS drives iNOS expression and NO production. (A) Presence of functional NADPH oxidase in DCs was tested by determining OCR in a Seahorse XF-24 analyzer in response to phorbol 12-myristate 13-acetate to activate the complex, and subsequently to specific NADPH oxidase inhibitor apocynin to block the complex. ${ }^{21}$ (B) DCs were seeded in a Seahorse XF-24 analyzer, stimulated with LPS or medium for 24 hours, and OCR was determined during sequential treatments with antimycin-A/rotenone (ETC inhibitors) and apocynin. Data represent means \pm SD of triplicates. One of 2 experiments is shown. (C) Relative NOS2 mRNA expression was determined in DCs 8 hours after medium or LPS stimulation. Data represent means \pm SD of 3 independent experiments. (D) Intracellular iNOS expression in 24-hour LPS-activated (black line) and unstimulated (gray histogram) DCs was determined by FACS. One experiment of 6 is shown. (E) iNOS expression was determined as in panel $\mathrm{D}$ at the indicated time points after LPS stimulation. Data represent means \pm SD 2 independent experiments. (F) Nitrite levels were determined in culture supernatants at the indicated time points after LPS stimulation. Data represent means \pm SD of 2 independent experiments. (G) DCs were seeded in a Seahorse XF-24 analyzer, stimulated with LPS or medium for 24 hours, and real-time OCR was determined during sequential treatments with antimycin-A/rotenone (ETC inhibitors) and the NOS inhibitor SEITU. Data represent means \pm SD of triplicates. One of 3 experiments is shown.

iNOS protein expression (Figure 2D) were readily detectable in LPS-activated DCs, but not in resting DCs. iNOS expression and concomitant NO production could also be detected to various degrees in DCs activated by agonists of TLR1/2, TLR3, TLR7/8, and TLR9 (supplemental Figure 2B-C). The induction of expression of iNOS (Figure 2E) and NO production (Figure $2 \mathrm{~F}$ ) coincided in time with the onset in loss of mitochondrial function (Figure 1A). Consistent with this, inhibition of iNOS with the potent NOS inhibitor SEITU reduced nonmitochondrial OCR in LPS-stimulated but not unstimulated cells (Figure 2G), indicating that most nonmitochondrial oxygen consumption in activated DCs is due to iNOS activity.

To address whether NO generated by iNOS underlies the loss of mitochondrial function, DCs were stimulated with LPS in the presence or absence of SEITU. SEITU treatment totally blocked NO production by DCs (Figure 3A). Strikingly, when iNOS function in LPS-stimulated DCs was inhibited, mitochondrial respiration, as assessed by extracellular flux analysis, was fully restored to the normal profile characteristic of functional mitochondria (Figure 3B). Likewise, LPS-activated $i \mathrm{NOS}^{-1-}$ DCs retained a normal OCR response profile (Figure 3C), $\Delta \Psi_{\mathrm{m}}$ (Figure 3D), and mitochondrial $\mathrm{O}_{2}{ }^{-}$production (Figure 3E). The inhibition of mitochondrial respiration in LPS-activated DCs was a direct effect of NO production by iNOS, because treatment of LPS-stimulated DCs in the presence of SEITU and an exogenous NO donor (SNAP) resulted in functional mitochondrial collapse (Figure 3F). Finally, the inhibitory effects of NO on OXPHOS were reversible, because SEITU treatment of DCs 24 hours after LPS stimulation restored mitochondrial respiration (Figure 3G), confirming that viable mitochondria persist in LPS-activated DCs for at least 24 hours.
Long-term increase in glycolytic metabolism is a response to the block in mitochondrial respiration to maintain cellular ATP levels and to prevent cell death

To determine whether the commitment to glycolytic metabolism in DCs in response to LPS stimulation ${ }^{4}$ is a direct result of the loss of mitochondrial respiration due to NO production, we analyzed the ECAR as an indication of lactate production and the glycolytic rate of DCs 24 hours after LPS stimulation in the presence or absence of SEITU. Consistent with our previous findings, ${ }^{4}$ LPS stimulation resulted in a significantly increased ECAR (Figure 4A). However, when iNOS was inhibited, LPS-activated DCs displayed an ECAR comparable to that of resting DCs (Figure 4A). The absence of an increase in glycolytic rate in LPS- and SEITU-treated DCs was confirmed by analysis of glucose consumption (Figure 4B) and lactate production (Figure 4C). The causal link between inhibition of OXPHOS and the increase in glycolytic rate was further substantiated by the finding that treatment of unstimulated DCs with drugs that interfere with mitochondrial respiration or with the NO donor SNAP resulted in an increase in ECAR (Figure 4D).

We showed previously that glucose deprivation or inhibition of glycolysis through the addition of an inhibitory glucose analog, 2-DG, inhibited the ability of DCs to become activated in response to LPS. ${ }^{4}$ On the basis of these results, we concluded that a sustained commitment to glycolytic metabolism is essential to allow DC activation. However, whereas glycolytic rates at 24 hours after activation were no higher than in resting cells when NO production was inhibited (Figure 4A-C), DC activation under these conditions was unimpaired or even enhanced, as determined by surface 
A

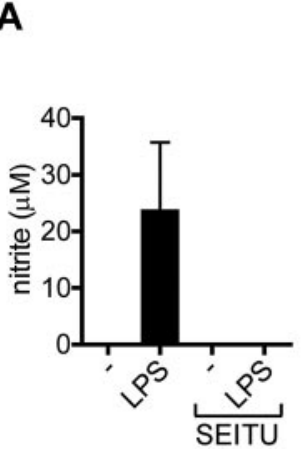

D

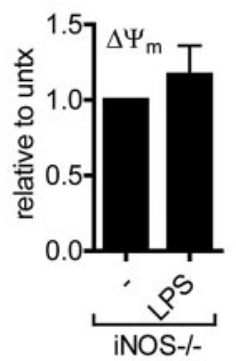

B

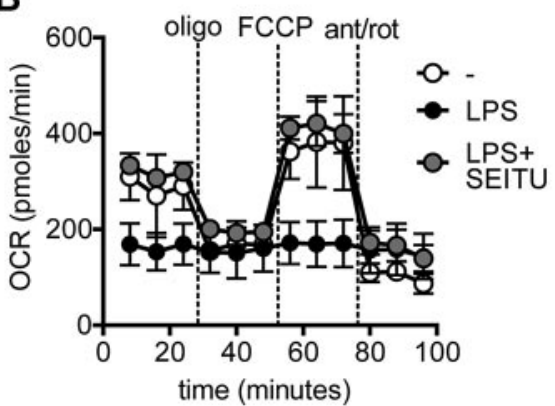

C

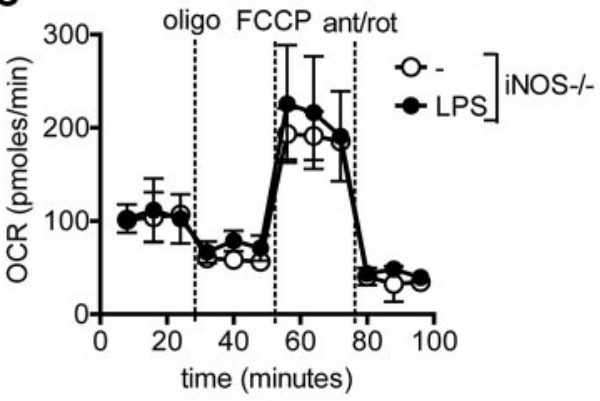

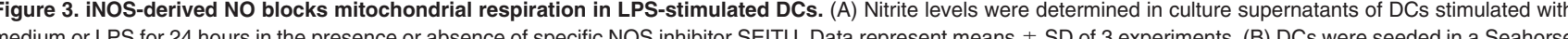
in the presence or absence of specific NOS inhibitor SEITU. Data represent means \pm SD of 3 experiments. (B) DCs were seeded in sequential treatments with oligomycin, FCCP, and antimycin-A/rotenone. Data represent means \pm SD of triplicates. One of 3 experiments is shown. (C) iNOS ${ }^{-1-}$ DCs were stimulated with medium or LPS for 24 hours and analyzed as in panel B. Data represent means \pm SD of triplicates. One of 3 experiments is shown. (D-E) Twenty-four-hour LPS-stimulated DCs were stained with hydroethidin to detect $\mathrm{O}_{2}^{-}$production or DiOC 6 to determine mitochondrial membrane potential $\left(\Psi_{\mathrm{m}}\right)$. Bars represent geoMFI $\pm \mathrm{SD}$ of 3 experiments and data are plotted relative to unstimulated cells. (F) DCs were stimulated for 24 hours with LPS in the presence or absence of SEITU and the NO donor SNAP as indicated, and analyzed as in panel B. Data represent means \pm SD of triplicates. One of 2 experiments is shown. (G) DCs were stimulated with medium, LPS for 24 hours, or LPS for 48 hours, with the last 24 hours in the presence of SEITU, and analyzed as in panel B. Data represent means \pm SD of triplicates and are shown as percentage of OCR before drug treatment. One of 2 experiments is shown. ant/rot indicates antimycin-A/rotenone; and oligo, oligomycin.

expression of MHC-I, MHC-II, and costimulatory molecules (Figure 4E), cytokine production (Figure 4F), and T cell-activating capacity (Figure 4G).

We also addressed the role of glycolysis in LPS-stimulated DCs that have lost mitochondrial function. We reasoned that in the absence of OXPHOS, DCs cannot generate ATP through mitochondrial respiration and thus would be dependent on glycolysis for ATP generation. Despite the absence of OXPHOS-derived ATP, LPS-stimulated DCs had higher cellular ATP levels than resting DCs (Figure 5A), suggesting that the increase in glycolytic rate in these cells generates sufficient ATP to more than compensate for the lack of mitochondrial ATP production. Confirmation that ATP was generated by glycolysis in these experiments was obtained by experiments in which glucose in DC cultures 24 hours after LPS activation was replaced by galactose to prevent a net ATP yield through glycolysis. ${ }^{23}$ Under these conditions, ATP levels decreased by more than $90 \%$ in 15 minutes, showing the heavy reliance of these cells on glycolysis for ATP production (Figure 5B). In contrast, in unstimulated DCs or LPS-stimulated $i \mathrm{NOS}^{-1-}$ DCs in which mitochondrial function is normal, galactose treatment resulted in only a 30\% reduction in ATP levels (Figure 5B). Most importantly, the generation of ATP through glycolysis was found to be essential for DC survival after LPS activation of wild-type, but not $\mathrm{NOS}^{-1-}$ DCs (Figure 5C). These data show that the increase in glycolytic metabolism in LPS-activated DCs is a direct consequence of a NO-induced blockade in OXPHOS and that the compensatory increase in glycolytic rate is essential for ATP generation to prevent cell death.

\section{Inflammatory moDCs display an iNOS-dependent block in mitochondrial respiration and require glycolysis for survival in vivo}

GM-CSF-cultured BM-derived DCs are considered a model for in vivo inflammatory moDCs that differentiate from circulating inflammatory monocytes in response to inflammation and infection. ${ }^{24}$ Inflammatory moDCs have been studied extensively during L monocytogenes infection, where they have been shown to express iNOS. ${ }^{11}$ To begin to address whether L monocytogeneselicited inflammatory moDCs display iNOS-dependent metabolic changes similar to those observed in activated BM-derived DCs, we isolated $\mathrm{CD} 11 \mathrm{c}^{+} \mathrm{CD} 11 \mathrm{~b}^{\text {int }} \mathrm{MHCII}{ }^{+} \mathrm{Ly} 6 \mathrm{C}^{\text {hi }}$ cells from the spleens of mice that had been infected with $L$ monocytogenes 2 days earlier and cultured them with and without LPS. Consistent with previous findings, ${ }^{11}$ a proportion of these inflammatory DCs expressed iNOS, which was increased in response to LPS exposure in vitro (Figure 6A). These cells also produced iNOS-derived NO immediately ex vivo, which was elevated in response to LPS (Figure 6B). In contrast, conventional DCs (cDCs) isolated from the spleens of naive mice did not express iNOS and could not be induced to do so by LPS stimulation (Figure 6A) or by stimulation with other TLR agonists or inflammatory cytokines (supplemental Figure 3). ${ }^{25}$ Most importantly, the OCR of LPS-activated inflammatory moDCs was largely nonresponsive to oligomycin, FCCP, and antimycinA/rotenone, but baseline OCR and responsiveness to these drugs was restored by the inhibition of NO synthesis (Figure 6C). Therefore, inflammatory moDCs exhibit a striking NO-dependent 
A

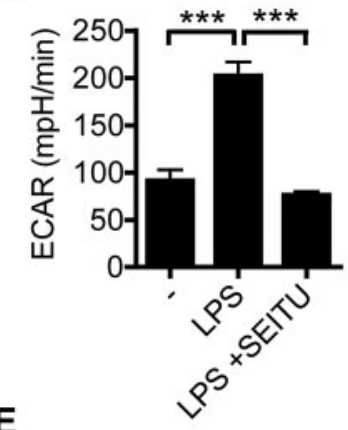

B

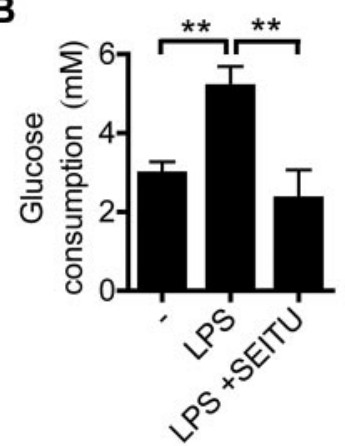

C

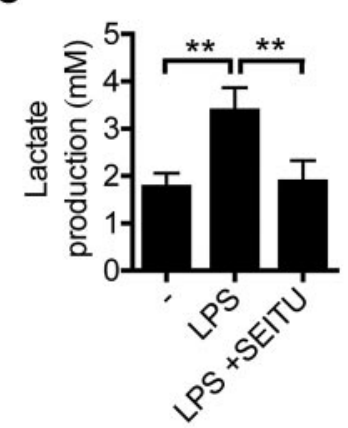

D

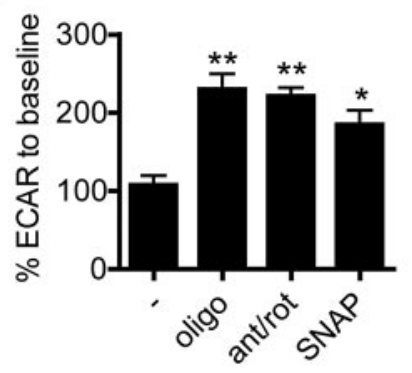

G

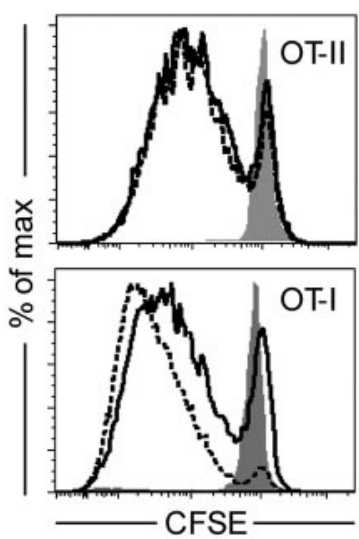

$\mathrm{TNF} \alpha$

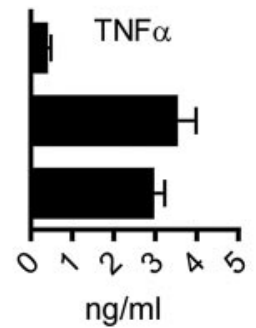

$\mathrm{ng} / \mathrm{ml}$

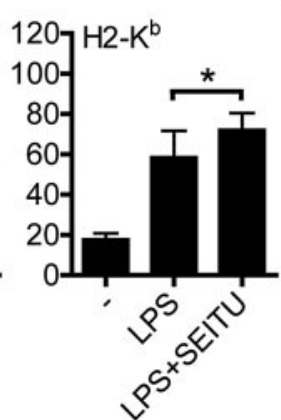

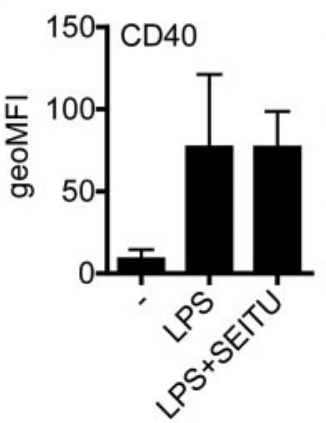

$\mathbf{F}$

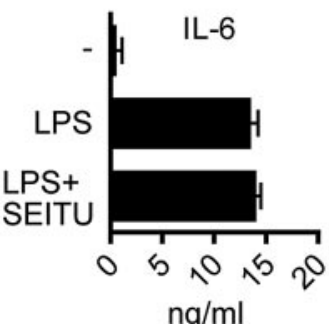

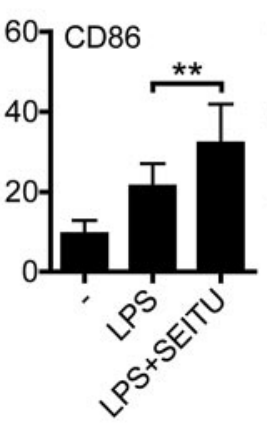

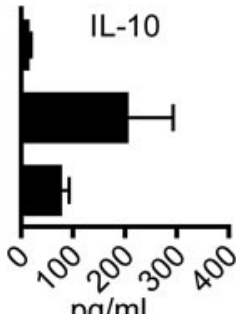

$\mathrm{pg} / \mathrm{ml}$
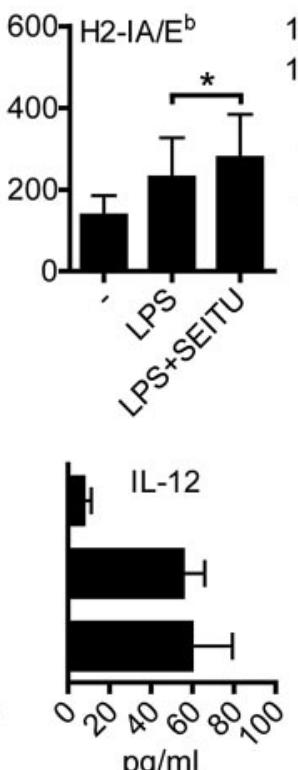

$\mathrm{pg} / \mathrm{ml}$

Figure 4. LPS-activated DCs commit to glycolysis in response to NO-induced inhibition of mitochondrial respiration, but do not require sustained glycolysis for normal activation. (A) DCs were seeded in a Seahorse XF-24 Analyzer and either left unstimulated or treated with LPS for 24 hours in the presence or absence of SEITU, after which real-time rates of ECAR as a readout for lactate production were determined. Data represent means \pm SD of 4 independent experiments. (B-C) DCs were treated as in panel $A$ and supernatants collected 24 hours later were used to determine glucose consumption (B) and lactate production (C). Data represent means \pm SD of 4 independent experiments. (D) DCs were seeded in a Seahorse XF-24 Analyzer and either left unstimulated or treated with the indicated reagents for 10 minutes, after which real-time rates of ECAR as a readout for lactate production were determined. Data represent means \pm SD of 3 independent experiments. (E-F) DCs were stimulated with LPS for 24 hours in the presence or absence of the NOS inhibitor SEITU after which surface expression of indicated markers was analyzed by FACS (E) or cytokine levels (F) were determined in supernatants. Data represent means \pm SD of 4 experiments. (G) Wild-type or iNOS ${ }^{-1-}$ DCs were treated for 6 hours with the indicated reagents and subsequently cultured for 4 days in a 1:10 ratio with CFSE-labeled naive OT-II or OT-I T cells. One of 3 experiments is shown. ${ }^{*} P<.05 ;{ }^{* *} P<.01 ;{ }^{* \star *} P<.001$. ant/rot indicates antimycin-A/rotenone; and oligo, oligomycin.

reduction in OXPHOS accompanied by an NO-dependent shift toward glycolytic metabolism that was not apparent in splenic cDCs (Figure 6D). Finally, given the importance of glycolysis for survival of iNOS-expressing DCs in vitro, we predicted that inflammatory moDCs would depend on glycolysis for survival in vivo. To investigate this, $L$ monocytogenes-infected mice were injected with 2-DG to block glycolysis and then the frequencies of splenic DC subsets were analyzed. Whereas $\mathrm{CD} 4^{+}$and $\mathrm{CD} 8 \alpha^{+}$ $\mathrm{cDC}$ and total inflammatory moDC frequencies were not altered by 2-DG treatment (Figure 6E), the frequency of iNOS-expressing inflammatory moDCs was significantly reduced (Figure 6F-G), supporting the notion that commitment to glycolysis is essential for the survival of iNOS-expressing DCs in vivo.

\section{Discussion}

DCs are a heterogeneous family of cells that are crucial in the initiation of inflammation and the induction of adaptive immune responses. ${ }^{26}$ A key feature that allows DCs to perform these functions is their ability to recognize and respond to pathogen- and danger-associated molecular patterns through receptor systems such as the TLRs. In the absence of TLR agonists, DCs are quiescent, but after TLR stimulation, the cells become activated and begin producing inflammatory mediators and expressing molecules that allow them to activate other cells within the immune system. ${ }^{2}$ We reported previously that activation of BM-derived DCs in response to TLR agonists is accompanied by a change in their metabolism, characterized by a persisting switch from OXPHOS to aerobic glycolysis, and found that inhibition of this process had profound negative effects on activation and cellular survival. ${ }^{4}$ We hypothesized that DCs commit to glycolysis because this is the only metabolic pathway able to support the metabolic and bioenergetic challenges of enacting the activation pathway. Using real-time metabolic assays, we showed in the present study that the early stage of DC activation in response to TLR agonists is accompanied by an increase in mitochondrial activity, but that this is transient and followed by functional mitochondrial collapse. We 
A

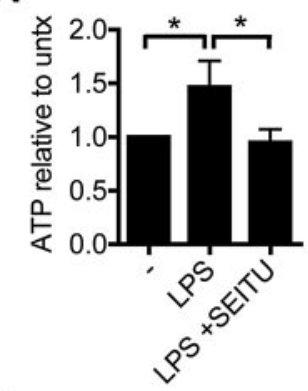

B

C
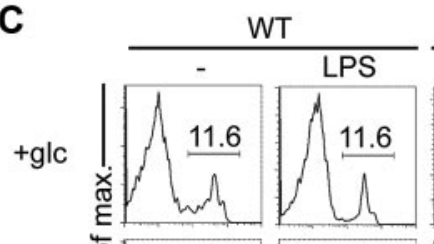
LPS + gal å fl the

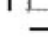
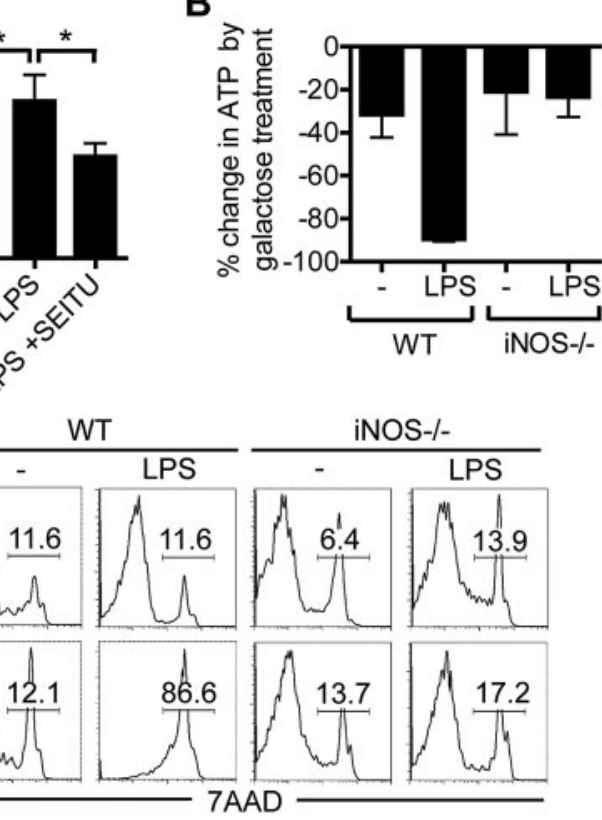

iNOS-I-

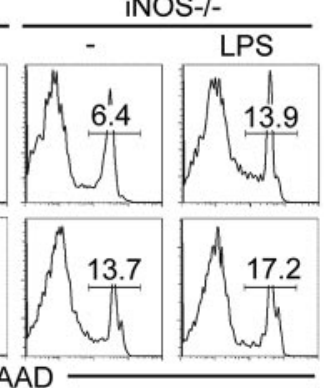

Figure 5. Commitment to glycolytic metabolism in LPS-activated DCs provides essential ATP for survival in the absence of mitochondrial respiration. (A) DCs were treated as indicated and 24 hours later cells were lysed and the relative ATP levels were measured. ATP data were normalized to ATP levels in unstimulated DCs. Data represent means $\pm S D$ of 4 independent experiments. (B) DCs were stimulated as indicated and 24 hours later, cells were cultured in medium in which glucose was replaced by galactose. After 15 minutes, cells were lysed and analyzed for ATP levels. ATP levels in DCs cultured in galactose-containing medium are shown as the percentage relative to DCs cultured in the presence of glucose. The percentage reduction in ATP levels represents ATP derived from glycolysis. Data represent means $\pm S D$ of duplicates of 1 of 2 independent experiments. (C) DCs were stimulated as in panel B for 24 hours and analyzed for cell death by 7-aminoactinomycin D staining after being cultured for an additional 24 hours in galactose- or glucose-containing medium. Data represent one of 2 independent experiments. ${ }^{*} P<.05$.

also showed that the onset of mitochondrial dysfunction coincides with the expression of TLR-driven iNOS expression and that endogenous NO production by this enzyme causes a block in mitochondrial respiration, resulting in a state that resembles hypoxia functionally. The commitment to glycolytic metabolism in activated DCs occurs only in DC subsets that make NO, is a direct consequence of the inhibition of OXPHOS by NO, and serves a vital survival function to provide ATP in the absence of mitochondrial ATP generation (Figure 7).

NO is a functionally pleiotropic molecule with roles in muscle contractility, platelet aggregation, neuronal activity, and immune responses. ${ }^{27}$ Endogenously produced NO has been shown to affect the microbicidal properties, ${ }^{11}$ activation, ${ }^{28}$ migration, ${ }^{29}$ and $\mathrm{T}$ cellpriming capacities ${ }^{30}$ of DCs. However, little is known about the effects of NO on DC metabolism. We have found that autocrine NO interferes with mitochondrial respiration directly in DCs. An autocrine inhibitory effect of NO on OXPHOS has been reported previously in astrocytes ${ }^{19}$ and macrophages. ${ }^{31} \mathrm{NO}$ is likely to inhibit OXPHOS by reversibly competing with oxygen to inhibit cytochrome $\mathrm{c}$ oxidase, the terminal enzyme of the ETC. ${ }^{17}$ Our observations that neither ATP synthase inhibition (by oligomycin) nor uncoupling of OXPHOS (by FCCP) induced any changes in oxygen consumption in TLR-activated DCs are consistent with NO acting directly on the ETC.

We found herein that after inhibition of OXPHOS by NO, LPS-activated DCs maintain energy production by up-regulating glycolysis, a phenomenon reminiscent of what has been observed in classically activated macrophages. ${ }^{32,33}$ In the absence of ATP production by glycolysis, intracellular ATP levels decreased precipitously within minutes, resulting in rapid cell death. Apart from providing ATP for energy-demanding processes such as translation and movement, glycolytic ATP is thought to be important for maintenance of $\Delta \Psi_{\mathrm{m}}$ in the absence of functional mitochondrial respiration. ${ }^{34}$ In this case, glycolytic ATP is consumed by ATP synthase acting in reverse to maintain a proton gradient across the inner mitochondrial membrane. This prevents depolarization of the mitochondria and thereby prevents release of cytochrome $\mathrm{c}$ and induction of apoptosis. ${ }^{23}$

The underlying mechanism(s) behind the up-regulation of glycolysis in response to inhibition of mitochondrial respiration remains to be identified. In astrocytes, NO can act through AMP-activated kinase to increase the activity of 6-phosphofructo2-kinase and induce expression of phosphofructo-2-kinase, both important positive regulators of glycolysis that were found to be essential for astrocyte survival in the presence of NO. ${ }^{35}$ Furthermore, glucose uptake in these cells was enhanced by NO via up-regulation of glucose transporter-3 in an AMP-activated kinasedependent manner. ${ }^{36}$ This may be analogous to the increased glucose transporter-1 expression observed in LPS-activated DCs. ${ }^{4}$ In addition, hypoxia-induced factor $1-\alpha(\mathrm{HIF}-1 \alpha)$, a key regulator of glycolysis during hypoxia, has been implicated in the upregulation of genes involved in glycolysis in response to $\mathrm{NO}$ in macrophages. ${ }^{31}$ However, the effects of NO on HIF-1 $\alpha$ stabilization seem to be different between normoxic and hypoxic conditions. ${ }^{37}$ An alternative view is that the NO-mediated inhibition of the ETC feeds back to block metabolic turnover in the mitochondrial TCA cycle, which results in an accumulation of pyruvate that is consequently converted into lactate to maintain glycolytic flow for ATP generation (Figure 7).

We showed previously that PI3K signaling downstream of TLR is required for the commitment to glycolytic metabolism. ${ }^{4}$ Together with the known importance of the PI3K signaling pathway in promoting glycolysis, ${ }^{8}$ this led us to propose that PI3K signaling in response to TLR ligation promotes the direct expression of genes supporting glycolytic metabolism. However, it is also known that PI3K signaling is crucial for iNOS expression and function. ${ }^{38,39}$ Therefore, the data presented herein suggest the possibility that the reported PI3K-driven long-term commitment to glycolysis after TLR ligation may be a consequence of iNOS expression and concomitant impairment of OXPHOS and not of the direct promotion of glycolysis itself.

We have proposed previously that commitment of DCs to glycolysis in response to TLR ligation is a reflection of the importance of this metabolic pathway for both DC activation and survival. ${ }^{4}$ However, our current findings suggest that the long-term switch to glycolysis in response to TLR ligation is essential for DC survival by providing ATP in the absence of OXPHOS, but is dispensable for DC activation, because no defect in activation was found of DCs in which iNOS was inhibited and in which no increase in long-term glycolysis was observed. In fact, our data (Figure 4E) indicate that NO-driven impairment of mitochondrial function results in reduced DC activation by TLR agonists. Together with the fact that DCs, when deprived of glucose during LPS stimulation, fail to mature, ${ }^{4}$ this leads us to propose that glycolysis may serve 2 purposes in DCs after TLR ligation. First, during the first few hours after TLR stimulation, glycolysis may be essential for providing the metabolic and bioenergetics resources required to enact the activation pathway. Second, once TLR ligation has induced iNOS expression, leading to an NO-mediated 
A

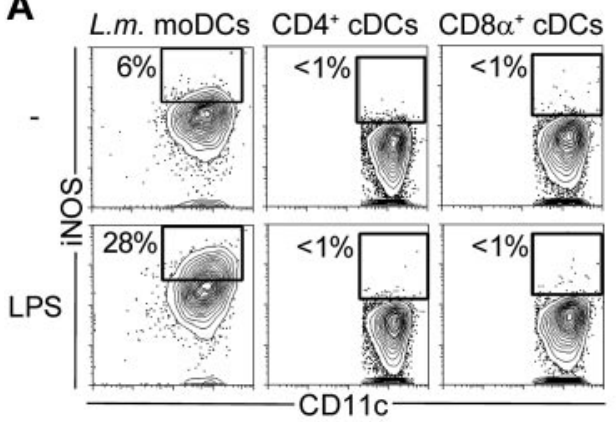

B

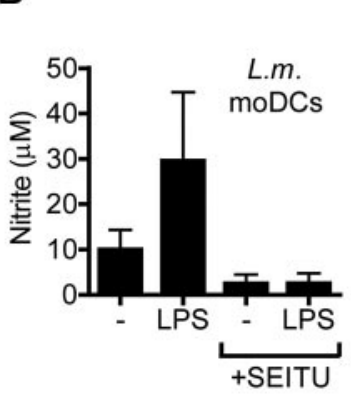

E

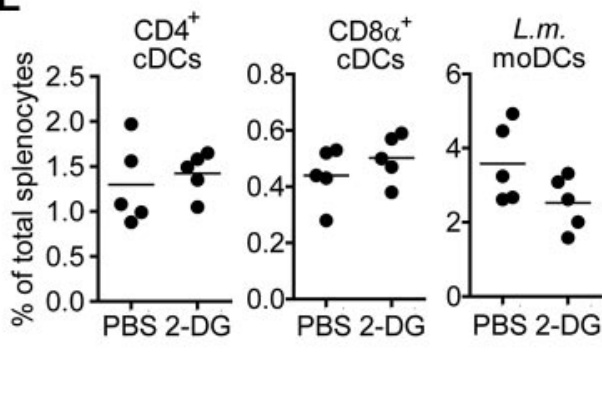

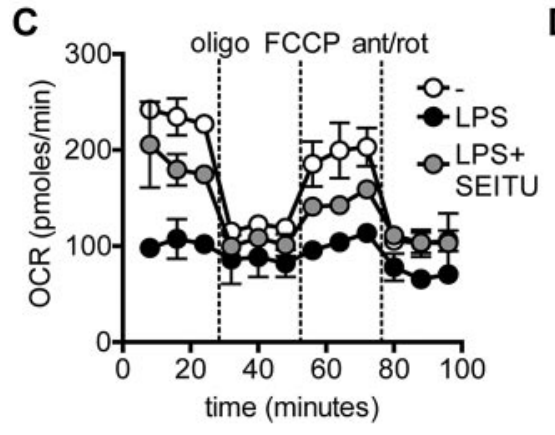
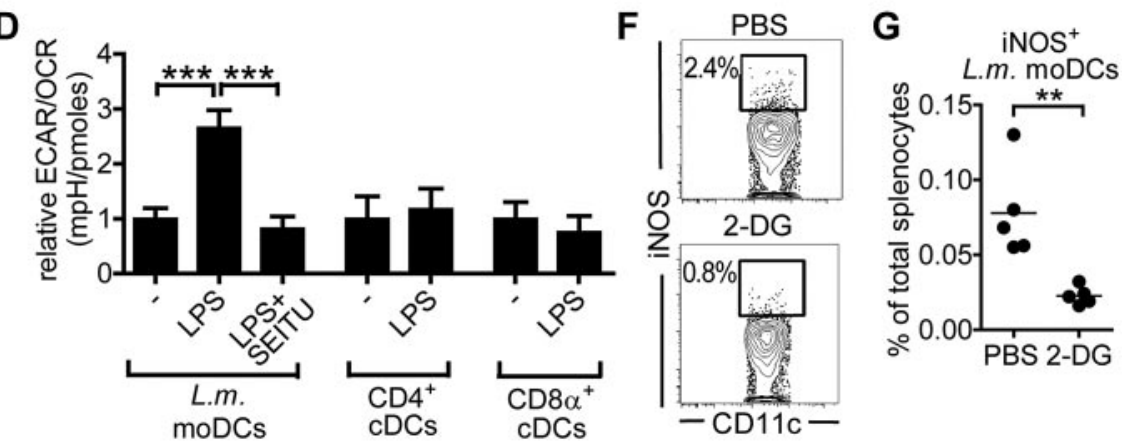

Figure 6. Ex vivo LPS-activated inflammatory moDCs display an iNOS-dependent block in mitochondrial respiration and depend on glycolysis for survival in vivo. Inflammatory splenic moDCs were isolated from mice infected for 2 days with $L$ monocytogenes (strain $\Delta$ ActA, $2 \times 10^{5}$ CFU, intravenous) by sorting by flow cytometry for

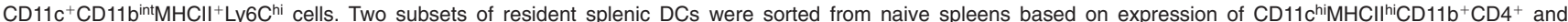
CD11 $\mathrm{c}^{\text {hi } M H C I I}{ }^{\text {hi }} \mathrm{DEC} 205^{+} \mathrm{CD} 8 \alpha^{+}$. (A) Splenic DC subsets were left unstimulated or stimulated ex vivo with LPS for 24 hours and subsequently analyzed for intracellular iNOS expression. NOS $^{-1-}$ cells were used to draw gates on iNOS ${ }^{+}$cells. Representative FACS plots of 1 of 3 experiments are shown. (B) Supernatants from cultures of 24 hours ex vivo-stimulated inflammatory DCs were analyzed for nitrite levels. Data represent means \pm SD of 3 experiments. (C) Ex vivo inflammatory moDCs were seeded in a Seahorse XF-24 analyzer, stimulated with medium or LPS for 24 hours in the presence or absence of SEITU, and real-time OCR was measured in response to sequential treatments with oligomycin, FCCP, and antimycin A/rotenone. Data represent means \pm SD of triplicates. One of 2 experiments is shown. (D) Splenic DC subsets were cultured and stimulated as in panel $C$ and the ratio between basal ECAR and OCR was calculated and plotted relative to the ratio of unstimulated DCs, which was set to 1. Data represent means \pm SD of triplicates. One of 2 experiments is shown. (E) Mice infected for 1 day with $L$ monocytogenes (strain $\Delta A c t A, 2 \times 10^{5} \mathrm{CFU}$, intravenous) were injected intraperitoneally with PBS or 2-DG (4 g/kg), and 6 hours later DC frequencies were determined in spleens. Data are representative of 5 individual mice per group. One of 2 independent experiments is shown. (F) Percentage of inflammatory moDCs staining positive for iNOS after PBS or 2-DG treatment as described in panel E. (G) Frequency of iNOS ${ }^{+}$inflammatory moDCs in spleens after PBS or 2-DG treatment as described in panel E. Data are representative of 5 individual mice per group. One of 2 independent experiments is shown. ${ }^{\star \star} P<.01 ;{ }^{\star \star \star} P<.001$.

loss of mitochondrial respiration, glycolysis may primarily provide ATP for cell survival. Furthermore, the observed increase in OCR during the first 6 hours in response to TLR ligation may hint at a role for mitochondrial metabolism in DC activation as well. We are currently trying to understand in detail how the early changes (within 0-6 hours of exposure to TLR agonists) in glycolytic and mitochondrial metabolism are regulated and how this affects DC activation and function.

An analysis of 3 different subsets of splenic DCs isolated from mice revealed a strong link between the ability to express iNOS and the commitment to sustained glycolysis after activation. Our data suggest that inflammatory moDCs, which do express iNOS, are
A

Figure 7. Schematic representation of iNOS-induced metabolic changes in DCs after TLR ligation. Unstimulated DCs can use both glycolysis and mitochondria for their bioenergetic and metabolic needs; however, TLR ligation induces iNOS expression and NO production that in an autocrine fashion inhibits the ETC and mitochondrial function. As a consequence, TLR-activated DCs enhance their glycolytic rate to prevent bioenergetic collapse and cell death.
B

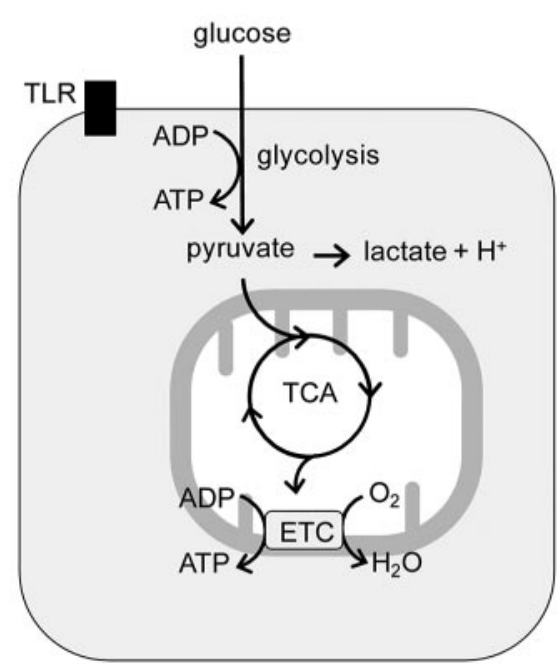

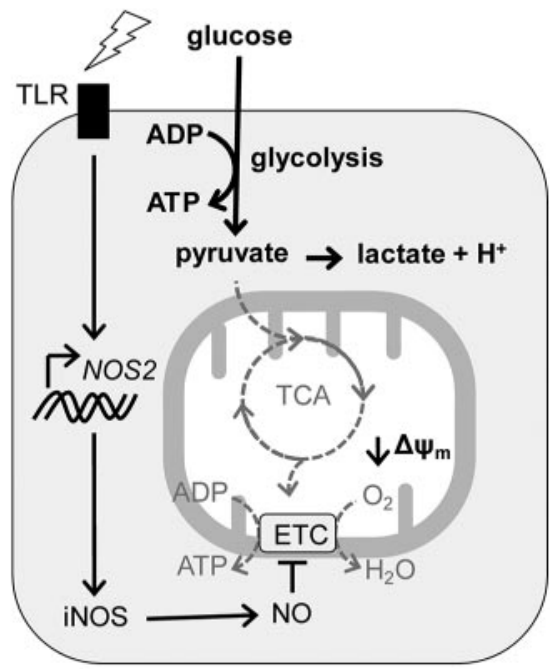


vulnerable to the same metabolic challenges faced by their in vitro-generated counterparts after activation. Only a small proportion (approximately 6\%) of the inflammatory moDCs expressed iNOS without TLR stimulation ex vivo, which was not sufficient to observe a significant effect on metabolism on the cultured cell population as a whole. Only when the DCs were restimulated ex vivo with LPS to induce iNOS expression in a higher percentage of cells was a switch to glycolytic metabolism observed. Whereas these observations do not address whether iNOS-dependent metabolic changes occur in DCs in vivo as well, our findings that glycolysis is specifically required for survival of iNOS-expressing DCs, but not other DC subsets in vivo, support the notion that individual iNOS-expressing DCs in vivo experience an autocrine NO-dependent block in mitochondrial respiration, resulting in a commitment to glycolysis that is essential for their survival. Apart from autocrine effects of NO, its production may affect the metabolism of adjacent cells as well. Given the known paracrine effects of NO derived from iNOS-expressing macrophages, ${ }^{40,41}$ it is possible that DC-derived NO affects the function of neighboring cells. For example, there is clear evidence from in vitro antigen-presentation assays that $\mathrm{NO}$ made by DCs is able to inhibit the proliferative ability of $\mathrm{T}$ cells, ${ }^{22,30}$ and it is interesting to speculate that this reflects an effect of NO on T-cell mitochondrial function.

Because inflammatory moDCs are thought to differentiate from monocytes at inflammatory sites, where oxygen levels can be low, their enhanced capacity to generate ATP anaerobically might be advantageous. Interestingly, not all cells are able to switch efficiently to glycolytic metabolism and to survive in response to impaired OXPHOS. ${ }^{42}$ It is conceivable that inflammatory moDCs are specialized to switch to glycolysis when necessary, precisely because they are able to make NO when activated and have evolved to enter potentially hypoxic environments. Therefore, it makes sense that they differ from resident lymphoid organ DCs, which are specialized to regulate T-cell responses to antigen arriving from distal sites. Regardless, our data imply that the ultimate effect on cellular survival and functionality of inhibition of the ETC by NO will depend on the availability of glucose to run the glycolytic pathway. Whereas the body has evolved to keep blood glucose levels within a narrow concentration range, local glucose concentrations in tissues, where highly activated inflammatory cells are infiltrating and competing for nutrients, are poorly understood. Further studies will be required to address these issues, and will help in the development of a clearer understanding of the functional consequences of iNOS-driven metabolic changes in DCs and other cells.

\section{Acknowledgments}

This work was supported by the National Institutes of Health (grant AI53825 to E.J.P.; grants RR000954, DK020579, and DK056341 to E.L.P. and K.E.Y.; and T32 training award AI049823 to E.A.), The Netherlands Organization for Scientific Research (Rubicon grant to G.J.W.W.), and the Trudeau Institute.

\section{Authorship}

Contribution: B.E. and E.A. designed the research, performed the experiments, and interpreted the data; G.J.W.v.d.W., R.C., K.E.Y., and E.L.P. helped with the experimental design and data interpretation; T.C.F. and R.C. performed the experiments; B.E. and E.J.P. wrote the manuscript; and E.J.P. designed and directed the research and interpreted the data.

Conflict-of-interest disclosure: The authors declare no competing financial interests.

Correspondence: E. J. Pearce, Division of Immunobiology, Department of Pathology and Immunology, Washington University School of Medicine, Campus Box 8118, 660 S Euclid Ave, St Louis, MO 63110; e-mail: edwardpearce@path.wustl.edu.

\section{References}

1. Kapsenberg ML. Dendritic-cell control of pathogen-driven T-cell polarization. Nat Rev Immunol. 2003;3(12):984-993.

2. Joffre O, Nolte MA, Sporri R, Reis e Sousa C. Inflammatory signals in dendritic cell activation and the induction of adaptive immunity. Immunol Rev. 2009;227(1):234-247.

3. Amati L, Pepe M, Passeri ME, Mastronardi ML, Jirillo E, Covelli V. Toll-like receptor signaling mechanisms involved in dendritic cell activation: potential therapeutic control of T cell polarization. Curr Pharm Des. 2006;12(32):4247-4254

4. Krawczyk CM, Holowka T, Sun J, et al. Toll-like receptor-induced changes in glycolytic metabolism regulate dendritic cell activation. Blood. 2010;115(23):4742-4749.

5. Warburg O. On the origin of cancer cells. Science. 1956;123(3191):309-314.

6. Shaw RJ. Glucose metabolism and cancer. Curr Opin Cell Biol. 2006;18(6):598-608.

7. Fox CJ, Hammerman PS, Thompson CB. Fuel feeds function: energy metabolism and the T-cell response. Nat Rev Immunol. 2005;5(11):844-852.

8. Cairns RA, Harris IS, Mak TW. Regulation of cancer cell metabolism. Nat Rev Cancer. 2011;11(2): 85-95.

9. Weinhouse $S$. The Warburg hypothesis fifty years later. Z Krebsforsch Klin Onkol Cancer Res Clin Oncol. 1976;87(2):115-126.

10. Lutz MB, Kukutsch N, Ogilvie AL, et al. An advanced culture method for generating large quan- tities of highly pure dendritic cells from mouse bone marrow. J Immunol Methods. 1999;223(1): 77-92.

11. Serbina NV, Salazar-Mather TP, Biron CA, Kuziel WA, Pamer EG. TNF/iNOS-producing dendritic cells mediate innate immune defense against bacterial infection. Immunity. 2003;19(1): 59-70.

12. Kimball E, Rabinowitz JD. Identifying decomposition products in extracts of cellular metabolites. Anal Biochem. 2006;358(2):273-280.

13. Guo W, Jiang L, Bhasin S, Khan SM, Swerdlow RH. DNA extraction procedures meaningfully influence qPCR-based mtDNA copy number determination. Mitochondrion. 2009;9(4):261-265.

14. Castera L, Hatzfeld-Charbonnier AS, Ballot C, et al. Apoptosis-related mitochondrial dysfunction defines human monocyte-derived dendritic cells with impaired immuno-stimulatory capacities. J Cell Mol Med. 2009;13(7):1321-1335

15. Wiegand G, Remington SJ. Citrate synthase: structure, control, and mechanism. Annu Rev Biophys Biophys Chem. 1986;15:97-117.

16. Kim I, Rodriguez-Enriquez S, Lemasters JJ. Selective degradation of mitochondria by mitophagy. Arch Biochem Biophys. 2007;462(2): 245-253.

17. Cleeter MW, Cooper JM, Darley-Usmar VM, Moncada S, Schapira AH. Reversible inhibition of cytochrome c oxidase, the terminal enzyme of the mitochondrial respiratory chain, by nitric oxide.
Implications for neurodegenerative diseases. FEBS Lett. 1994;345(1):50-54.

18. Ago T, Kuroda J, Pain J, Fu C, Li H, Sadoshima J. Upregulation of Nox4 by hypertrophic stimuli promotes apoptosis and mitochondrial dysfunction in cardiac myocytes. Circ Res. 2010;106(7):12531264.

19. Bolaños JP, Peuchen S, Heales SJ, Land JM, Clark JB. Nitric oxide-mediated inhibition of the mitochondrial respiratory chain in cultured astrocytes. J Neurochem. 1994;63(3):910-916.

20. Khan SA, Nanduri J, Yuan G, et al. NADPH oxidase 2 mediates intermittent hypoxia-induced mitochondrial complex I inhibition: relevance to blood pressure changes in rats. Antioxid Redox Signal. 2011;14(4):533-542.

21. Elsen S, Doussiere J, Villiers CL, et al. Cryptic O2-generating NADPH oxidase in dendritic cells. J Cell Sci. 2004;117(Pt 11):2215-2226.

22. Lu L, Bonham CA, Chambers FG, et al. Induction of nitric oxide synthase in mouse dendritic cells by IFN-gamma, endotoxin, and interaction with allogeneic T cells: nitric oxide production is associated with dendritic cell apoptosis. J Immunol. 1996;157(8):3577-3586.

23. Garedew A, Henderson SO, Moncada S. Activated macrophages utilize glycolytic ATP to maintain mitochondrial membrane potential and prevent apoptotic cell death. Cell Death Differ. 2010; 17(10):1540-1550.

24. Xu Y, Zhan Y, Lew AM, Naik SH, Kershaw MH. 
Differential development of murine dendritic cells by GM-CSF versus Flt3 ligand has implications for inflammation and trafficking. J Immunol. 2007; 179(11):7577-7584.

25. Powell TJ, Jenkins CD, Hattori R, MacPherson GG Rat bone marrow-derived dendritic cells, but not ex vivo dendritic cells, secrete nitric oxide and can inhibit T-cell proliferation. Immunology. 2003; 109(2):197-208.

26. Hashimoto D, Miller J, Merad M. Dendritic cell and macrophage heterogeneity in vivo. Immunity. 2011;35(3):323-335.

27. Gao Y. The multiple actions of NO. Pflugers Arch. 2010;459(6):829-839.

28. Adler HS, Simon A, Graulich E, et al. Neuronal nitric oxide synthase modulates maturation of human dendritic cells. J Immunol. 2010;184(11): 6025-6034.

29. Sugita K, Kabashima K, Yoshiki R, et al. Inducible nitric oxide synthase downmodulates contact hypersensitivity by suppressing dendritic cell migration and survival. J Invest Dermatol. 2010;130(2): 464-471.

30. Hoffman RA, Mahidhara RS, Wolf-Johnston AS, Lu L, Thomson AW, Simmons RL. Differential modulation of CD4 and CD8 T-cell proliferation by induction of nitric oxide synthesis in antigen pre- senting cells. Transplantation. 2002;74(6):836845.

31. Garedew A, Moncada S. Mitochondrial dysfunction and HIF1alpha stabilization in inflammation. J Cell Sci. 2008;121(Pt 20):3468-3475.

32. Albina JE, Mastrofrancesco B. Modulation of glucose metabolism in macrophages by products of nitric oxide synthase. Am J Physiol. 1993;264 (6 Pt 1):C1594-C1599.

33. Mateo RB, Reichner JS, Mastrofrancesco B, Kraft-Stolar D, Albina JE. Impact of nitric oxide on macrophage glucose metabolism and glyceraldehyde-3-phosphate dehydrogenase activity. Am J Physiol. 1995;268(3 Pt 1):C669-675.

34. Beltrán B, Mathur A, Duchen MR,

Erusalimsky JD, Moncada S. The effect of nitric oxide on cell respiration: a key to understanding its role in cell survival or death. Proc Natl Acad Sci U S A. 2000;97(26):14602-14607.

35. Almeida A, Moncada S, Bolanos JP. Nitric oxide switches on glycolysis through the AMP protein kinase and 6-phosphofructo-2-kinase pathway. Nat Cell Biol. 2004;6(1):45-51.

36. Cidad P, Almeida A, Bolanos JP. Inhibition of mitochondrial respiration by nitric oxide rapidly stimulates cytoprotective GLUT3-mediated glucose uptake through $5^{\prime}$-AMP-activated protein kinase. Biochem J. 2004;384(3):629-636.
37. Agani FH, Puchowicz M, Chavez JC, Pichiule P, LaManna J. Role of nitric oxide in the regulation of HIF-1alpha expression during hypoxia. $\mathrm{Am}$ J Physiol Cell Physiol. 2002;283(1):C178-C186.

38. Sakai K, Suzuki H, Oda H, et al. Phosphoinositide 3-kinase in nitric oxide synthesis in macrophage: critical dimerization of inducible nitric-oxide synthase. J Biol Chem. 2006;281(26):17736-17742.

39. Kao SJ, Lei HC, Kuo CT, et al. Lipoteichoic acid induces nuclear factor-kappaB activation and nitric oxide synthase expression via phosphatidylinositol 3-kinase, Akt, and p38 MAPK in RAW 264.7 macrophages. Immunology. 2005;115(3): 366-374.

40. Brown GC, Foxwell N, Moncada S. Transcellular regulation of cell respiration by nitric oxide generated by activated macrophages. FEBS Lett. 1998;439(3):321-324.

41. Stuehr DJ, Nathan CF. Nitric oxide. A macrophage product responsible for cytostasis and respiratory inhibition in tumor target cells. J Exp Med. 1989;169(5):1543-1555.

42. Almeida A, Almeida J, Bolanos JP, Moncada S. Different responses of astrocytes and neurons to nitric oxide: the role of glycolytically generated ATP in astrocyte protection. Proc Natl Acad Sci U S A. 2001;98(26):15294-15299. 